\title{
Measurement of Beta-Function and Phase Using the Response Matrix*
}

\author{
Y. Chung, G. Decker and K. Evans, Jr. \\ Argonne National Laboratory, Argonne, IL 60439
}

\begin{abstract}
A new method for extracting the beta-function and phase for the beam position monitors (BPMs) and the corrector magnets from the measured response matix is presented. The response matrix relates beam motion at the BPM locations to changes in corrector magnet strengths. Using the model beta and phase as the initial values, new values are obtained by iteration. The accuracy of beta and phase thus calculated is limited by the accuracy of response matrix measurement and calibration of BPMs and correctors. The scaling ambiguity in the beta-function is resolved by matching the beta product and phase advance across a drift region. A by-product of this technique is an accurate determination of the betatron tune, and in principle, quadrupole strengths can be calculated from the betas and phases. This method is applied to data obtained from the X-ray ring at the National Synchrotron Light Source at Brookhaven. The possibility of applying the results to lattice-debugging will also be discussed.
\end{abstract}

\section{INTRODUCTION}

In the past, several methods for the measurement of accelerator beta functions and betatron phase have been suggested and applied. Accelerators having independently controllable quadrupole magnets can make use of the variation of tune with quadrupole magnet strength to extract the value of beta at the location of the quad, as is done at the Cornell Electron Storage Ring [1]. A technique was suggested by Harrison and Peggs [2] whereby betas and phases could be determined from closed orbit measurements resulting from varying two steering correctors in turn, provided that the values of beta and phase at those two correctors could be found from some other technique. The beta function and phase at the locations of all beam position monitors (BPMs) could in principle be found in this way.

In this paper, a technique is presented for determining the best fit betas and phases at all BPMs and all steering correctors from measured response matrix data. The response matrix relates beam motion at the BPM locations to changes in steering corrector strengths. This technique is similar to that of Ref. [2], but makes use of all steering correctors rather than just two. Because the problem is severely overconstrained, the method of singular value decomposition (SVD) of a matrix is used to minimize the difference between the resulting ring model and the measured response matrix data. One by-product of this technique is an accurate

*Work supported by the U.S. Department of Energy, Office of Basic Energy Sciences, under Contract No. W-31-109-ENG-38 determination of the betatron tune. The technique is applied to data obtained from the $\mathrm{X}$-ray ring at the National Synchrotron Light Source at Brookhaven. Results are obtained which agree qualitatively with measurements made there two and a half years ago using the technique of Ref. [2], together with the least squares fitting, by Decker and Swenson. [3]

\section{THEORY}

Suppose that there are M BPMs and N correctors in the storage ring. Both $M$ and $N$ are larger than 1 . Let $\beta_{i}$ and $\psi_{i}$ be the amplitude and phase functions at the location of the $\mathrm{i}$-th BPM. $\beta_{c j}$ and $\psi_{c j}$ are similarly defined for the $j$-th corrector. The response matrix $R_{i j}$ corresponding to the beam motion at the $i$-th BPM per unit angle of kick by the $j$-th corrector is then given by [4]

$$
\mathrm{R}_{\mathrm{ij}}=\frac{\sqrt{\beta_{i} \beta_{\mathrm{cj}}}}{2 \sin \pi \nu} \cos \left(\psi_{i}-\psi_{\mathrm{cj}} \pm \pi v\right) . \quad\left\{\begin{array}{l}
+: \psi_{i}<\psi_{\mathrm{cj}} \\
-: \psi_{i}>\psi_{\mathrm{cj}}
\end{array}\right.
$$

$v$ is the betatron tune of the machine. The response matrix $R_{i j}$ can be obtained from measurements, and we will develop in the following a method to extract the beta function and phase from the measured data using Eq. (1).

\section{A. Calculation of Beta and Phase Functions}

Let us define

$$
\psi_{c i j}= \begin{cases}\psi_{c j}, & \psi_{c j}<\psi_{i} \\ \psi_{c j}-2 \pi v . & \psi_{c j}>\psi_{i}\end{cases}
$$

Then it can be shown that $\beta_{i}$ and $\psi_{i}$ are given by

$$
\begin{gathered}
\beta_{i}=\left(\mathrm{c}_{\mathrm{i}}^{2}+\mathrm{s}_{\mathrm{i}}^{2}\right) \sin ^{2} \pi v, \\
\Psi_{i}=\tan ^{-1}\left(\frac{\mathrm{s}_{i}}{\mathrm{c}_{\mathrm{i}}}\right)+\pi v, \quad\left(0<\psi_{\mathrm{i}}<2 \pi \nu \text { and } \Psi_{\mathrm{i}+1}>\psi_{\mathrm{i}}\right)
\end{gathered}
$$

where $c_{i}$ and $s_{i}$ are solutions of

$$
c_{i} \cos \psi_{c i j}+s_{i} \sin \psi_{c i j}=\frac{2}{\sqrt{\beta_{c j}}} R_{i j} . \quad(1 \leq j \leq N)
$$

In matrix form, Eq. (4) can be written as

$$
\mathbf{A} \cdot \mathbf{x}=\mathbf{a}
$$

\section{MASTRR}

The submitted manuscript has been authored by a contractor of the U.S. Government under contract No. W.31-109ENG-38. nonexclusive, royalty-free license to publish or reproduce the published form of this contribution, or allow others to do for contribution, or allow others to do so, for 
where

$$
\mathbf{A}=\left(\begin{array}{cc}
\cos \Psi_{\mathrm{cil}} & \sin \Psi_{\mathrm{cil}} \\
: & : \\
\cos \Psi_{\mathrm{ciN}} & \sin \psi_{\mathrm{ciN}}
\end{array}\right), \quad \mathbf{x}=\left(\begin{array}{c}
\mathrm{c}_{\mathrm{i}} \\
\mathrm{s}_{\mathrm{i}}
\end{array}\right)
$$

and

$$
a=\left(\begin{array}{c}
\frac{2 R_{i 1}}{\sqrt{\beta_{c l}}} \\
\vdots \\
\frac{2 R_{i N}}{\sqrt{\beta_{c N}}}
\end{array}\right) .
$$

The functions $\beta$ and $\psi$ for the correctors can be similarly obtained. With the definition

$$
\psi_{i j}= \begin{cases}\psi_{i}, & \Psi_{i}<\psi_{c j} \\ \psi_{i}-2 \pi v, & \Psi_{i}>\psi_{c j}\end{cases}
$$

beta and phase functions for the correctors can be obtained from

$$
\begin{gathered}
\beta_{\mathrm{cj}}=\left(c_{\mathrm{cj}}{ }^{2}+s_{\mathrm{cj}}{ }^{2}\right) \sin ^{2} \pi \nu, \\
\Psi_{\mathrm{cj}}=\tan ^{-1}\left(\frac{s_{\mathrm{cj}}}{c_{\mathrm{cj}}}\right)+\pi \nu . \quad\left(0<\Psi_{\mathrm{cj}}<2 \pi \nu \text { and } \Psi_{\mathrm{cj}+1}>\Psi_{\mathrm{cj}}\right)
\end{gathered}
$$

$c_{c j}$ and $s_{c j}$ are solutions of

$$
\mathbf{B} \cdot \mathbf{y}=\mathbf{b} \text {, }
$$

where

$$
\mathbf{B}=\left(\begin{array}{cc}
\cos \psi_{1 j} & \sin \Psi_{1 j} \\
: & : \\
\cos \Psi_{M j} & \sin \psi_{M j}
\end{array}\right), \quad y=\left(\begin{array}{c}
c_{c j} \\
s_{c j}
\end{array}\right) \text {, }
$$

and

$$
\mathbf{b}=\left(\begin{array}{c}
\frac{2 R_{1 i}}{\sqrt{\beta_{1}}} \\
: \\
\frac{2 R_{M i}}{\sqrt{\beta_{M}}}
\end{array}\right) .
$$

In Eq. (5), $A$ is an $N \times 2$ matrix which is a function of the phases of both the BPMs and correctors. $x$ and a depend on the BPMs and the correctors, respectively. Similarly, in Eq. (10), $B$ is an $M \times 2$ matrix which is a function of the phases of both the BPMs and correctors. $y$ and $b$ depend on the correctors and the BPMs, respectively. In addition, $v$ is also an unknown parameter to be determined.
In order to obtain a self-consistent set of solutions, we will use iteration. We start with a set of initial values for $\left\{\beta_{i}, \psi_{i}\right\}$, $\left\{\beta_{c j}, \psi_{c j}\right\}$, and $v$, which we use to obtain the matrices $\mathbf{A}$ and $\mathbf{B}$ and the vectors $\mathbf{a}$ and $\mathbf{b}$. Since the number of equations is larger than the number of unknowns, the problem is overdetermined, and therefore, the exact solution does not exist in general. However, approximate solutions can still be obtained such that $|\mathbf{A} \cdot \mathbf{x}-\mathbf{a}|$ and $|\mathbf{B} \cdot \mathbf{y}-\mathbf{b}|$ have the minimum values possible. These are the closest solutions for Eqs. (5) and (10), and can be obtained using the technique of singular value decomposition (SVD). [5] The solutions thus obtained will best fit the measured response matrix and are used as the initial guess for the next iteration. This process continues until the solutions converge for a given $v$.

\section{B. Error Analysis and Determination of Tune}

With the solutions for $\beta$ and $\psi$, the response matrix $\mathbf{R}^{\prime}$ is reconstructed using Eq. (1) and the r.m.s. error

$$
\Delta R=\sqrt{\frac{1}{M N} \sum_{i, j}\left(R_{i j}^{\prime}-R_{i j}\right)^{2}}
$$

is calculated. The error function $\Delta R$ represents the accuracy of the tune $v$ used for iteration. The correct tune corresponds to the minimum $\Delta R$, which again can be used to estimate the error in the $\beta$-function. Let us assume that the relative error in $\beta$ is constant. That is,

$$
\frac{\delta \beta_{\mathrm{i}}}{\beta_{\mathrm{i}}} \approx \frac{\delta \beta_{\mathrm{cj}}}{\beta_{\mathrm{cj}}}=\delta \mathrm{a}=\mathrm{constant} \text { for all } \mathrm{i}, \mathrm{j} \text {. }
$$

Then from Eq. (1), it can be shown

$$
\delta \mathrm{a} \leqslant 4 \sin (\pi v) \Delta \mathrm{R}<\beta_{\mathrm{i}} \beta_{\mathrm{cj}}>^{-1 / 2} .
$$

$<\cdots>$ denotes the average value. This analysis, however, does not include the calibration errors in the BPMs and correctors, which could actually dominate the measurement error.

\section{Resolution of Beta-Scaling Ambiguity}

Inherent in the preceding analysis are the ambiguities in the scaling of beta functions and the offset of the phase function. The phase offset has no physical meaning and we will concentrate on the resolution of beta-scaling ambiguity.

In Eq. (1), $R_{i j}$ remain unchanged when $\beta_{\mathrm{i}}$ is multiplied and $\beta_{\mathrm{cj}}$ is divided by a constant. Therefore, an extra constraint is necessary to determine the beta functions for BPMs and correctors. Consider a drift region of length $L$ which has beta functions $\beta_{1}$ and $\beta_{2}$ at the end points and phase difference of $\Delta \psi$. Then we have

$$
\sqrt{\beta_{1} \beta_{2}} \sin \Delta \psi=\mathrm{L}
$$

If a pair of BPMs or correctors can be found which have drift region between them, the scaling constant for the beta functions can be determined using Eq. (16). Multiple such pairs could be used to check for errors. 


\section{Measurement of Quadrupole Strength}

The strength of a quadrupole magnet can be determined if beta and phase functions are known at points on both sides as shown in Fig. 1 . The point 1 is at a distance $l_{1}$ from the quadrupole magnet and has $\beta_{1}$ and $\psi_{1}$; similarly for point 2 on the other side. The quadrupole has length $l_{q}$.

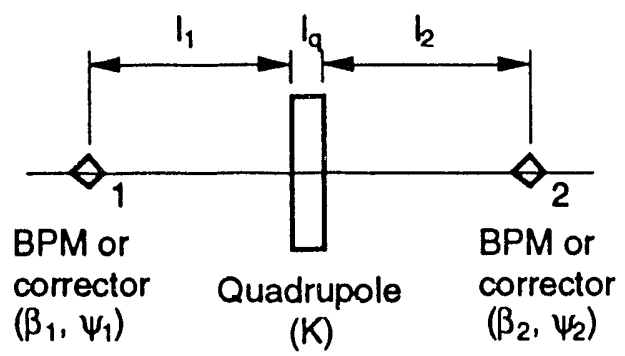

Fig. 1: Determination of quadrupole strength using $\beta$ and $\psi$.

The quadrupole strength $\mathrm{K}$ is then determined from

$$
\begin{aligned}
& \sqrt{\beta_{1} \beta_{2}} \sin \left|\psi_{2}-\psi_{1}\right|= \\
& \begin{cases}\left(\frac{1}{q}-l_{1} l_{2} q\right) \sin \left(q l_{q}\right)+\left(l_{1}+l_{2}\right) \cos \left(q l_{q}\right), & K>0 \\
\left(\frac{1}{q}+l_{1} l_{2} q\right) \sinh \left(q l_{q}\right)+\left(l_{1}+l_{2}\right) \cosh \left(q l_{q}\right), & K<0\end{cases}
\end{aligned}
$$

where $q=\sqrt{|K|}$. In case the right-hand side of Eq. (17) is not very sensitive to changes in $q$, the accuracy of the solution depends largely on that of the $\beta$ and $\psi$ functions.

\section{ANALYSIS OF NSLS X-RAY RING}

In this section, we analyze the response matrix in the vertical plane of the X-ray ring of the National Synchrotron Light Source (NSLS) at Brookhaven National Lab. [6] Fortysix BPMs and 39 correctors were used.

With $=0.005 \mathrm{~mm}$ error of BPM reading and corrector kick of $\approx 0.16 \mathrm{mrad}$ for measurement of the response matrix, the measurement error $\delta R$ is estimated to be $\delta R \approx 0.005 / 0.16 \approx$ $3 \times 10^{-2}(\mathrm{~m} / \mathrm{rad})$. Figure 2 shows the plot of $\Delta R$ as a function of $v$. The minimum point is $v=6.184$, where $\Delta R=2.4 \times 10^{-2}$ $\mathrm{m} / \mathrm{rad}$. This is in good agreement with the estimated error $\delta R$.

The $\beta$-functions obtained from the measurement $(v=$ $6.184)$ and the ring model $(v=6.2)$ are shown in Fig. 3. From Eq. (15), the relative error $\delta a$ in calculation of the $\beta$-functions is less than $2.3 \times 10^{-3}$.

When the results were applied to obtain the strengths of the quadrupoles, significant deviations $(\approx 40 \%)$ from the nominal values were observed. This is largely attributable to (1) calibration error in the BPMs and correctors and (2) the fact that $\mathrm{K}$ is very sensitive to the resulting error in $\beta$-functions $(\Delta K / K \approx 0.3 \Delta \beta(m)$ ). Considering the proximity of adjacent magnets to the correctors in the ring, sensitivity calibration of the correctors seems to be the dominant error source.

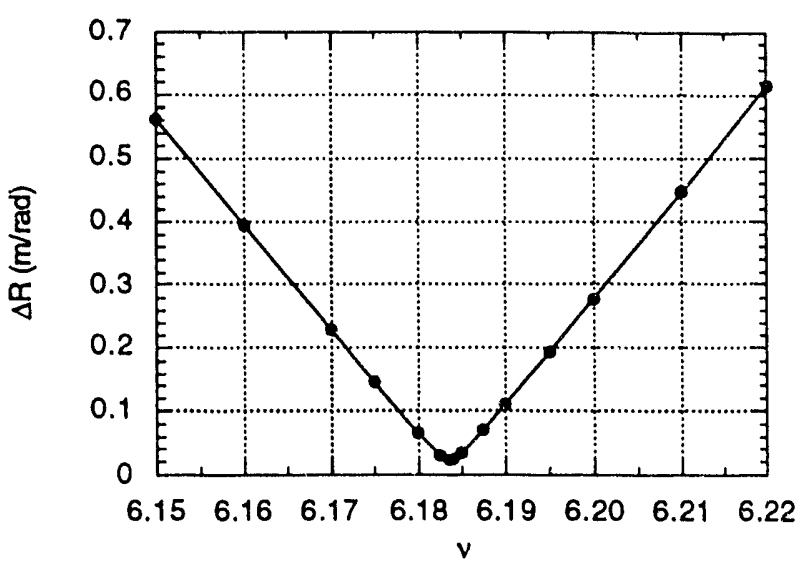

Fig. 2: Response matrix error to determine the vertical tune of the NSLS X-ray ring. The minimum point is: $v=6.184, \Delta R=$ $2.4 \times 10^{-2} \mathrm{~m} / \mathrm{rad}$. The nominal tune is 6.2 .

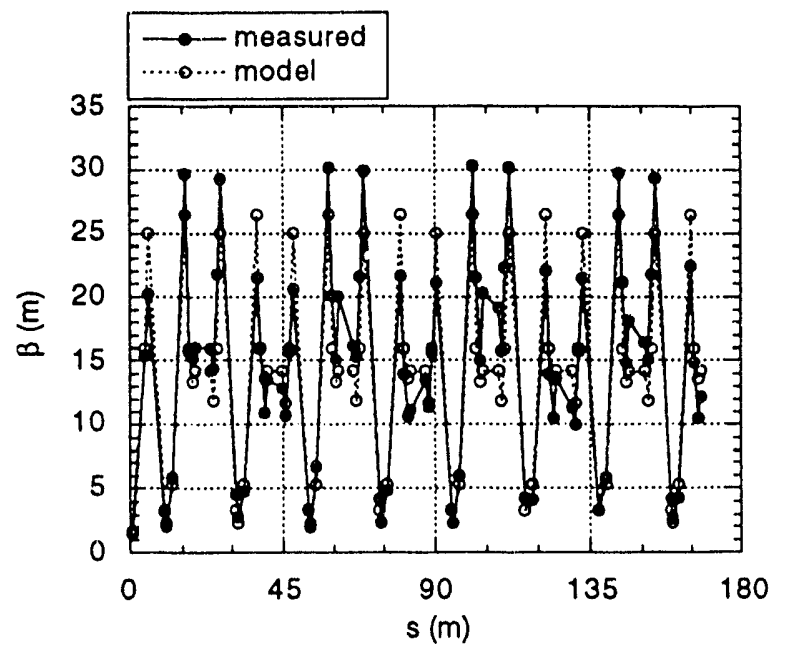

Fig. 3: The model and measured $\beta$-functions of the NSLS Xray ring in the vertical plane.

\section{REFERENCES}

[1] G. Decker, "Operational Aspects of Experimental Accelerator Physics," LS-182, Argonne National Lab, 1992.

[2] M. Harrison and S. Peggs, "Global Beta Measurement from Two Perturbed Closed Orbits," Proc. 1987 Particle Accelerator Conference, IEEE CH2387-9/87/0000-1105

[3] G. Decker and C. Swenson, unpublished.

[4] M. Sands, "The Physics of Electron Storage Rings - An Introduction," SLAC.-121, 1970.

[5] W. Press et al., Numerical Recipes in $C$, Cambridge University Press, p. 60, 1989.

[6] J. Safranek, private communication. 


\section{DISCLAIMER}

This report was prepared as an account of work sponsored by an agency of the United States Government. Neither the United States Government nor any agency thereof, nor any of their employees, makes any warranty, express or implied, or assumes any legal liability or responsibility for the accuracy, completeness, or usefulness of any information, apparatus, product, or process disclosed, or represents that its use would not infringe privately owned rights. Reference herein to any specific commercial product, process, or service by trade name, trademark, manufacturer, or otherwise does not necessarily constitute or imply its endorsement, recommendation, or favoring by the United States Government or any agency thereof. The views and opinions of authors expressed herein do not necessarily state or reflect those of the United States Government or any agency thereof. 

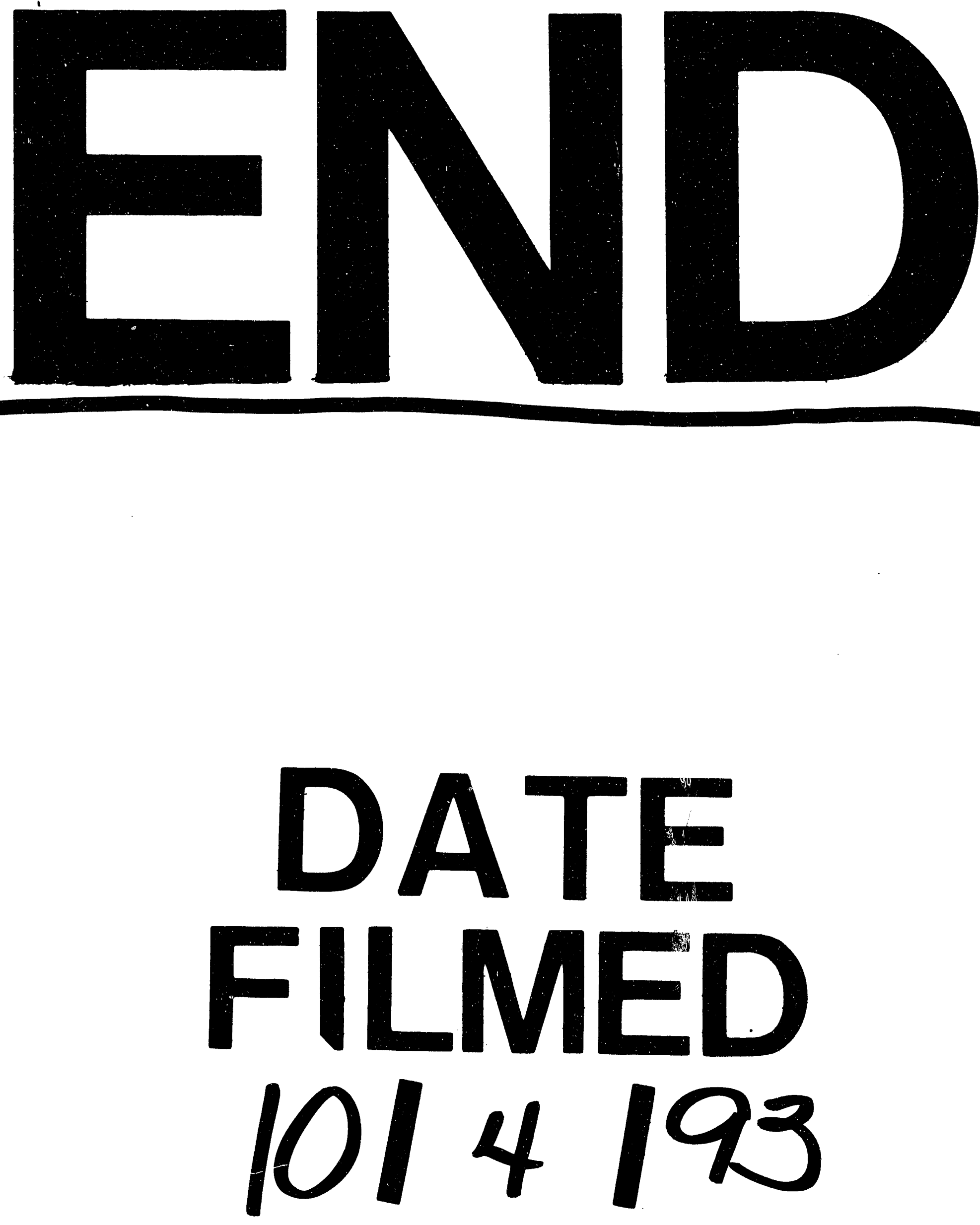
